\title{
From Convergent Beam Electron Diffraction to 4D-STEM: New opportunities for revealing structure at the atomic scale
}

Joanne Etheridge ${ }^{1}$, Wei Chao ${ }^{2}$, Bryan Esser ${ }^{2}$, Weilun $\mathrm{Li}^{3}$, Harkirat Mann ${ }^{1}$, Timothy Petersen ${ }^{2}$ and Changlin Zheng ${ }^{4}$

${ }^{1}$ Monash University, Monash University, Victoria, Australia, ${ }^{2}$ Monash University, United States, ${ }^{3}$ Monash University, Monash University, Australia, ${ }^{4}$ Fudan University, United States

Over the last half century, convergent beam electron diffraction (CBED) [1,2] has been established as an important technique for acquiring specimen information, such as thickness, lattice parameters, strain, space group, structure factor amplitudes [3] and phases [4,5] and bonding charge density distributions [6]. This information is generated via multiple, dynamical electron scattering and is stored in the angulardependence of the scattered intensity distribution, which is acutely-sensitive to structure and bonding.

CBED is at the heart of every scanning transmission electron microscopy experiment (STEM). In STEM, an electron beam is scanned across the specimen, and at each position of the electron probe, a CBED pattern strikes the STEM detector in the back-focal plane. Conventional, fast read-out STEM detectors are in the shape of a disc or annulus, offering extremely limited angular resolution, thus 'losing' much of the detailed specimen information available in the CBED pattern. More recently, relatively slow but highly pixelated detectors have enabled the whole CBED pattern to be recorded with much higher angular resolution [7-10]. This powerful new capability provides access to much more of the information available in the CBED pattern. Furthermore, it enables this information to be measured from each probe position and mapped across the specimen. This is currently known as a 4D-STEM experiment [11] but, as pixelated detectors increase in speed, it will likely become the standard operating mode and just called "STEM".

This new capability is being exploited in many ways, from the measurement of polarity to the study of beam sensitive materials to pushing the limits of resolution [11].

In this talk, we will discuss how to harvest selected parts of the CBED pattern to generate 'images' that are sensitive to the information we are seeking about a specimen. This includes counting atoms, identifying surface ad-atoms, measuring local displacements of atoms, mapping specimen symmetry and imaging defects using symmetry information [12]. This is illustrated with applications to functional materials, including plasmonic nanoparticles and functional perovskites and oxides.

\section{Acknowledgements}

The authors acknowledge the use of the instruments and scientific and technical assistance at the Monash Centre for Electron Microscopy, a Node of Microscopy Australia. This research was enabled by Australian Research Council grants LE0454166, DP150104483 and DP160104679 and DP200103070.

References

1. M. Tanaka, M. Terauchi, K. Tsuda, K. Saitoh: Convergent Beam Electron Diffraction Vols I to IV JEOL Ltd (2002)

2. J.C.H. Spence and J.M. Zuo Electron Microdiffraction: Plenum, New York (1991)

3. J. Gjønnes International Tables for Crystallography (2006). Vol. C. ch. 8.8, pp. 735-743 (2006) 
4. Nakashima, PH, Moodie, AF, Etheridge, J Proceedings of the National Academy of Sciences 110 14144-14149 (2013)

5. Guo Y, Nakashima P, Etheridge J IUCrJ 5 753-764 (2018)

6. JCH Spence Acta Cryst. A49, 231-260 (1993)

7. Battaglia M, et al. (2010) Nuclear Instruments and Methods in Physics Research Section A: Accelerators, Spectrometers, Detectors and Associated Equipment 622(3):669-677.

8. Ballabriga R, et al. (2011) Nuclear Instruments and Methods in Physics Research Section A: Accelerators, Spectrometers, Detectors and Associated Equipment 633:S15-S18.

9. Ryll H, et al. (2014) Microscopy and Microanalysis 20(S3) 1122-1123.

10. Tate MW, et al. (2016) Microscopy and Microanalysis 22 237-24 (2016).

11. C. Ophus Microscopy and Microanalysis 25 563-582 (2019)

12. Krajnak M, Etheridge J Proceedings of the National Academy of Sciences 117 27805-27810 (2020) 